\title{
Aggressive Asian tiger mosquito invades Europe
}

A

$\mathrm{n}$ aggressive mosquito that carries dengue and chikungunya fever is reproducing throughout Europe after being imported from Asia, with potentially devastating public health impacts.

The Asian tiger mosquito, or Aedes albopictus, is an invasive species native to Southeast Asia. It has spread rapidly to other parts of the world via shipments of goods such as used tires infested with mosquito eggs or through vegetation or water containers transported in aircraft, boats and vehicles that unknowingly harbour larvae or eggs, according to a report by the European Environmental Agency.

The mosquito, named for the black and white stripes on its body, is a vector for 20 diseases, including yellow fever, Rift Valley fever, West Nile virus and Japanese encephalitis virus. Although researchers don't know how many of these diseases the mosquito can transmit to humans, "there is solid evidence for its role in the transmission of two diseases: dengue and chikungunya," according to the agency's report.

The report, The Impacts of Invasive Alien Species in Europe (www.eea.europa .eu/publications/impacts-of-invasive-alien -species), links the mosquito to an outbreak of chikungunya fever affecting about 150 people in northeastern Italy in 2007. The agency also credits the Asian tiger mosquito with causing outbreaks of chikungunya on La Reunion Island in 2005-2006 that resulted in more than 250000 infections and several deaths.

The outbreak of chikungunya in Italy "has clearly shown that the tiger mosquito is an important disease vector and may represent a true public health challenge," the report says.

Chikungunya is characterized by the acute onset of fever (often with a rash), followed by severe pain in multiple joints for weeks to several months. Some studies report patients developing chronic joint pain three years after the onset of the illness.

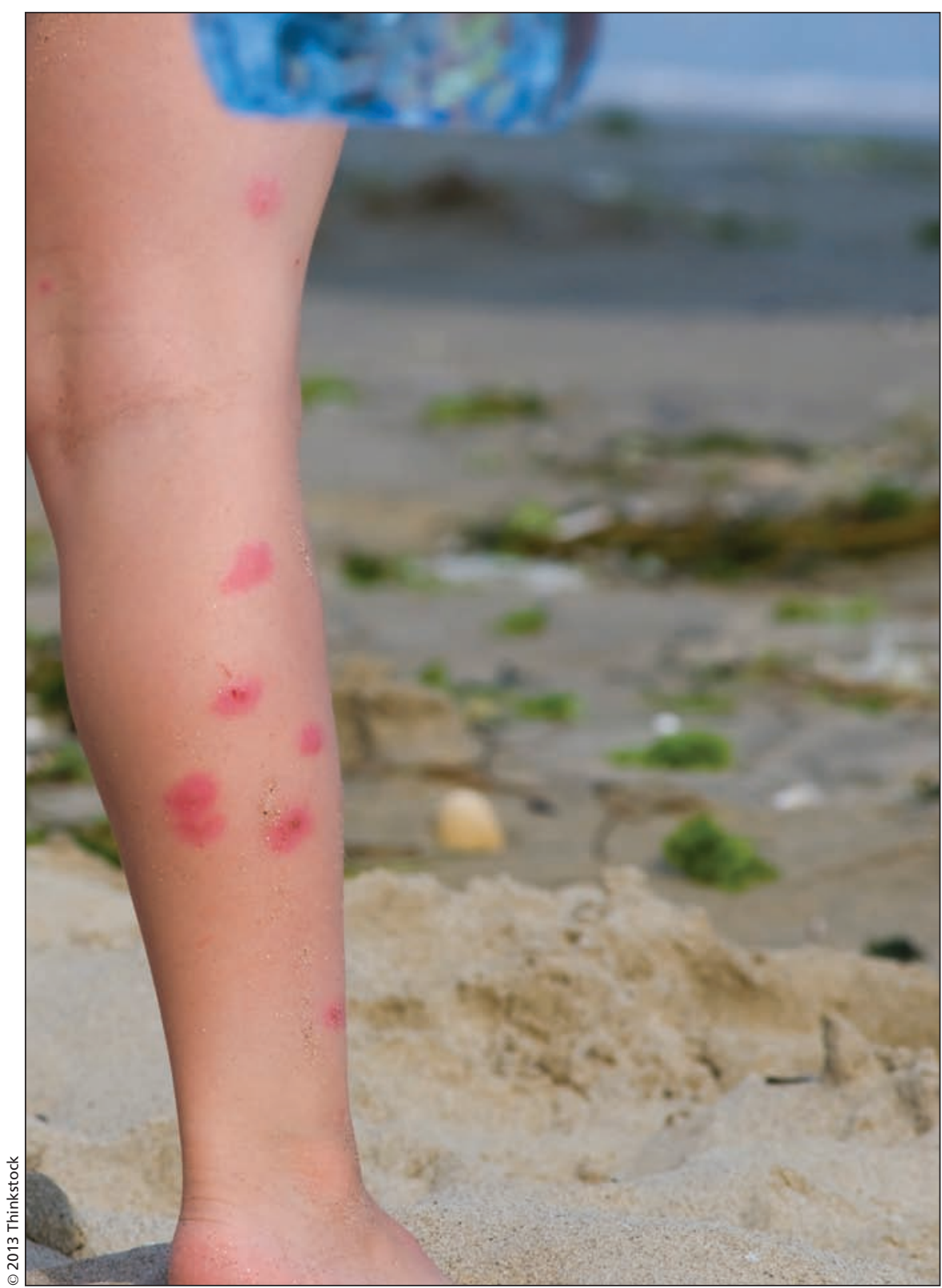

The tiger mosquito is a vector for 20 diseases, including yellow fever, Rift Valley fever, West Nile virus and Japanese encephalitis virus.

A year before the outbreak in Italy, India experienced a large outbreak of chikungunya in which about 500 people contracted the virus every day in a dozen states during July through September.

The European Environmental Agency is concerned that the tiger mosquito will soon be found in other parts of Europe as well, including the Baltic states and Scandinavia. Established populations of the tiger mosquito are already present in Albania, Bosnia and Herzegovina, Croatia, France (including Corsica), Greece, Monaco, Montenegro, the Netherlands, Italy, Slovenia, Spain and Switzerland.

"The scale of the challenge may be growing," Jacqueline McGlade, executive 
director of the European Environmental Agency, told $C M A J$ via email. "Climate change projections show the tiger mosquito will likely spread further, particularly in the Mediterranean basin, but also further north."

Raising public awareness about the health risks the tiger mosquito poses is crucial to reducing the public health impact the insect represents, says Piero Genovesi, coauthor of the report and the senior scientist with the Institute for Environmental Protection and Research. For example, people accustomed to mosquitoes that usually bite in the late afternoon or at night need to know that the Asian tiger mosquito bites during the day.

There are other more systemic prevention measures importers and exporters can also take.

"We now know that just drying used tires before importing them would have significantly reduced the risk of causing the outbreak, and [of introducing] chikungunya and dengue to Europe," Genovesi told CMAJ in an email.

"Invasive species cost Europe over 12.5 billion euros per year, and a significant proportion of these costs would have been prevented by applying effective policies," adds Genovesi, who is also the chair of the International Union for Conservation of Nature's Invasive Species Specialist Group.

"We can learn much from the health system on how to address invasions, improving our ability to identify potential invaders before they arrive to our territories, or at the earliest possible stage of invasions, when it is much less costly and complex to effectively address the new invaders." Soumyadeep Bhaumik, MBBS, Kolkata, India

CMAJ 2013. DOI:10.1503/cmaj.109-4462 